Chronic Obstructive Pulmonary Diseases: Journal of the COPD Foundation

\author{
Journal Club
}

\title{
Journal Club-Severe Acute Respiratory Syndrome Coronavirus-2: Impact on COPD Patients
}

\author{
Ron Balkissoon, MD, MSc, DIH, FRCPC ${ }^{1}$
}

Abbreviations: coronavirus disease 2019, COVID-19; severe acute respiratory coronavirus 2, SARS-CoV-2; intensive care unit, ICU; inhaled corticosteroid, ICS; angiotensin converting enzyme II, ACE-2; transmembrane protease Serine 2, TMPRSS2; long-acting beta-2 agonist, LABA; long-acting muscarinic antagonists, LAMA

Citation: Balkissoon R. Journal club-Severe acute respiratory syndrome coronavirus-2: impact on COPD patients. Chronic Obstr Pulm Dis. 2020;7(4):413-419. doi: https://doi.org/10.15326/jcopdf.7.4.2020.0188

\section{Denver, Colorado}

\section{Address correspondence to:}

Ron Balkissoon, MD, MSc, DIH, FRCPC

balkissoonr@njhealth.org

\section{Keywords}

coronavirus disease-2019; COVID-19; chronic obstructive pulmonary disease; COPD; severe acute respiratory syndrome coronavirus-2; SARS-CoV-2

\section{Introduction}

As of October 11, 2020, there have been 7,694,865 documented cases and 213,614 deaths attributed to coronavirus 2019 (COVID-19) disease from severe acute respiratory syndrome coronavirus-2 (SARSCoV-2) infection in the United States. ${ }^{1}$ Globally, the figures are $37,347,102$ cases and 1,074,732 deaths respectively. ${ }^{2}$ COVID-19 patients typically develop a cough and fever and approximately 20\% of patients develop shortness of breath within 8 days of symptom onset and $10 \%$ of patients will present with pulmonary infiltrates. Adult respiratory distress syndrome develops in about $25 \%$ of patients who get admitted to the hospital around 10.5 days after onset. $^{3,4}$ Early reports suggested that high risk groups included older individuals, males, and patients with underlying cardiovascular disease, diabetes and obesity. It was somewhat perplexing that patients with underlying respiratory illnesses did not seem to be at greater risk, given that the predominant symptom manifestations of the disease were respiratory. There are several probable reasons why gathering such data has been challenging such as, lack of testing and case identification early on, underdiagnosis of COPD patients in the general population at large and the fact that presumed susceptible patients, (such as those with asthma or COPD), were often told to stay home and isolate and were never tested. Hence, the prevalence rates that have been reported to date for COPD and/or asthma patients, largely reflect hospitalized and intensive care unit (ICU) cohorts and range from $1.1 \%$ to $38 \%{ }^{5,6}$ Needless to say, it is almost certain that the actual prevalence in patients with COPD has been underestimated in most studies. Guan et al reported that in a cohort of 1590 patients with COVID-19 in China, patients who had a diagnosis of COPD had an odds ratio of 2.681 (95\% CI 1.424-5.048; $P=0.002$ ) for ICU admission, mechanical ventilation or death compared to patients without any respiratory illness. ${ }^{7,8}$ They also found that $62.5 \%$ of severe cases had COPD compared with only $15.3 \%$ in non-severe cases. Furthermore, 25\% of those who died had COPD whereas of those who survived, only $2.8 \%$ had been diagnosed with COPD. Further studies that prospectively identify patients with COPD at time of evaluation for SARS-CoV-2 are needed to obtain more accurate estimates of the prevalence of COPD patients with evidence of infection. Nonetheless, it appears to be clear that COPD patients who develop infection have a higher risk of poor outcomes.

Some investigators have proposed that the reported low prevalence of COPD patients diagnosed with COVID-19 might reflect a potential protective effect of inhaled corticosteroids (ICSs). Attaway et al ${ }^{9}$ 
assembled a large cohort of 15,586 symptomatic patients at the Cleveland Clinic who were tested for COVID-19. Of those, $9.2 \%$ had COPD. Adjusting for covariates, they found no significant differences in the rate of COVID-19 positivity between COPD and nonCOPD patients. They did note significantly higher rates of hospitalization, (adjusted odds ratio of 1.36), ICU admissions (adjusted odds ratio 1.2) and invasive mechanical ventilation (adjusted odds ratio 1.49) in the COPD patients compared to non-COPD patients. They also found that patients who tested positive for COVID-19 were 2.4 times less likely to have used ICSs at the time of testing than those who tested negatively $(18.3 \%$ versus $44.8 \%, P<0.001) .{ }^{9}$ There has been ongoing debate regarding the relative benefit and risks of ICSs in patients with COPD particularly with regard to evidence suggesting an increased risk of lower respiratory tract infections. ${ }^{10}$ Recent studies have shown exposure to ICSs correlated with lower expression of angiotensin converting enzyme II (ACE-2) and transmembrane protease Serine 2 (TMPRSS 2) receptors, the entry receptors used by SARS-CoV-2 in sputum cells. ${ }^{11}$ Others have shown that ICSs may suppress SARS-CoV-2 replication ${ }^{11}$ or inhibit the production of cytokines in cells exposed to other human coronaviruses. ${ }^{12}$ Oral dexamethasone has also been shown to reduce the risk of death in people with severe COVID-19. ${ }^{13,14}$ Interestingly, there have been studies that suggested that the risk of lower respiratory tract infections may be related to the dose of the ICS used, suggesting that lower dose ICSs may be optimally efficacious and safest. ${ }^{15-19}$ The question remains: should we try to minimize ICSs because of the potential risk of rendering COPD patients more susceptible to infection or should we increase the dose to exploit potential protective effects?

Hence, there remain ongoing questions about the actual prevalence of COPD in COVID-19 patients and what factors may render COPD patients more susceptible to more severe disease. Also, there remain controversies regarding the benefits versus risks of ICSs in patients with COPD. In this Journal Club I examine some of the more recent papers that have addressed these issues.

Note: Abstracts are presented in their original published format and have not been edited to match JCOPDF style

\section{Abstract 1 \\ Prevalence, Severity and Mortality Associated with COPD and Smoking in Patients with COVID-19: A Rapid Systematic Review and Meta-Analysis}

Alqahtani JS, Oyelade T, Aldhahir AM, et al. Plos One. 2020;15(5):e0233147

doi: https://doi.org/10.1371/journal.pone.0233147

Background: Coronavirus disease 2019 (COVID-19) is an evolving infectious disease that dramatically spread all over the world in the early part of 2020. No studies have yet summarized the potential severity and mortality risks caused by COVID-19 in patients with chronic obstructive pulmonary disease (COPD), and we update information in smokers.

Methods: We systematically searched electronic databases from inception to March 24, 2020. Data were extracted by two independent authors in accordance with the Preferred Reporting Items for Systematic Reviews and Meta-Analyses guidelines. Study quality was assessed using a modified version of the Newcastle-Ottawa Scale. We synthesized a narrative from eligible studies and conducted a metaanalysis using a random-effects model to calculate pooled prevalence rates and $95 \%$ confidence intervals $(95 \% \mathrm{CI})$.

Results: In total, 123 abstracts were screened, and 61 full-text manuscripts were reviewed. A total of 15 studies met the inclusion criteria, which included a total of 2473 confirmed COVID-19 patients. All studies were included in the meta-analysis. The crude case fatality rate of COVID-19 was $7.4 \%$. The pooled prevalence rates of COPD patients and smokers in COVID-19 cases were $2 \%$ (95\% CI, $1 \%-$ $3 \%)$ and $9 \%(95 \%$ CI, $4 \%-14 \%)$ respectively. COPD patients were at a higher risk of more severe disease (risk of severity $=63 \%,(22 / 35)$ compared to patients without COPD 33.4\% (409/1224) [calculated RR, 1.88 (95\% CI, 1.4-2.4)]. This was associated with higher mortality (60\%). Our results showed that $22 \%(31 / 139)$ of current smokers and $46 \%$ (13/28) of ex-smokers had severe complications. The calculated RR showed that current smokers 
were 1.45 times more likely [95\% CI: $1.03-2.04$ ] to have severe complications compared to former and never smokers. Current smokers also had a higher mortality rate of $38.5 \%$.

Conclusion: Although COPD prevalence in COVID-19 cases was low in current reports, COVID-19 infection was associated with substantial severity and mortality rates in COPD. Compared to former and never smokers, current smokers were at greater risk of severe complications and higher mortality rate. Effective preventive measures are required to reduce COVID-19 risk in COPD patients and current smokers.

\section{Comments}

This was the first systematic review to look at the prevalence of SARS-CoV-2 cases specifically in COPD patients as well as in smokers and non-smokers. Of the 15 studies that met inclusion criteria 14 of them were from China $(\mathrm{n}=2452)$ and 1 from the United States ( $n=21$ patients). As noted in the Introduction, the prevalence rate in COPD patients was relatively low at $58 / 2473$ (2.3\%). The studies included in this analysis had relatively small sample sizes with 13 of the 15 studies having fewer than 200 patients (range 211099). Only 6 of the studies had data on severe cases and only 3 studies reported on COPD survival and death rates specifically. Another important omission is the lack of data on comorbidities often associated with COPD given their potential impact on severity of illness and mortality. Nonetheless, the finding that patients with COPD and current smokers appear to be at greater risk of severe complications certainly validates the concern for the risk to COPD patients of becoming infected and requires further research to help clinicians and patients establish effective prevention strategies. The findings also highlight the need for further research to find the mechanisms that render COPD patients and current smokers at higher risk for more severe disease and higher mortality.

\section{Abstract 2 \\ Underlying Respiratory Diseases, Specifically COPD, and Smoking are Associated With Severe COVID-19 Outcomes: A Systematic Review and Meta-Analysis}

Sanchez-Ramirez DC, Mackey D. Respir Med. 2020; 171:106096.

doi: https://doi.org/10.1016/j.rmed.2020.106096

Background: An outbreak of Corona Virus Disease 2019 (COVID-19) has spread rapidly reaching over 3 million of confirmed cases worldwide. The association of respiratory diseases and smoking, both highly prevalent globally, with COVID-19 severity has not been elucidated. Given the gap in the evidence and the growing prevalence of COVID-19, the objective of this study was to explore the association of underlying respiratory diseases and smoking with severe outcomes in patients with COVID-19 infection.

Methods: A systematic search was performed to identify studies reporting prevalence of respiratory diseases and/or smoking in relation with disease severity in patients with confirm COVID-19, published between January 1 to April 15, 2020 in English language. Pooled odds-ratio (OR) and 95\% confidence intervals (95\% CI) were calculated.

Findings: Twenty-two studies met the inclusion criteria. All the studies presented data of 13,184 COVID-19 patients (55\% males). Patients with severe outcomes were older and a larger percentage were males compared with the non-severe. Pooled analysis showed that prevalence of respiratory diseases (OR 4.21; 95\% CI, 2.9-6.0) and smoking (current smoking OR 1.98; 95\% CI, 1.16-3.39 and former smoking OR 3.46; 95\% CI, 2.46-4.85) were significantly associated with severe COVID-19 outcomes.

Interpretation: Results suggested that underlying respiratory diseases, specifically COPD, and smoking were associated with severe COVID-19 outcomes. These findings may support the planning 
of preventive interventions and could contribute to improvements in the assessment and management of patient risk factors in clinical practice, leading to the mitigation of severe outcomes in patients with COVID-19 infection.

\section{Comments}

This systematic review and meta-analysis of 22 studies included 1 Centers for Disease Control study of the U.S. population (7162 patients) and the remaining studies were from China with a total patient cohort of 13,184 . The remaining 21 studies reported the prevalence of respiratory diseases and included 12,976 patients. This study is significant for not only demonstrating an association between the increased risk for severe disease in patients who are current smokers but also for former smokers. Furthermore, this study also indicated an increased risk for patients with underlying chronic respiratory diseases. COPD was the main respiratory disease documented in the studies. There was a higher prevalence of underlying respiratory disease (12\%) found in patients with severe COVID-19 outcomes compared with the non-severe outcomes group (4\%). These are similar to the findings of Attaway et al mentioned above. ${ }^{9}$ Hopefully, the results from these studies assist in the development of intervention programs aimed at prevention and improving patient assessment and management. Further research is also required to look at the causal mechanisms underlying these observed associations.

\section{Abstract 3 \\ Distribution of ACE2, CD147, CD26, and Other SARS-CoV-2 Associated Molecules in Tissues and Immune Cells in Health and in Asthma, COPD, Obesity, Hypertension, and COVID-19 Risk Factors}

Radzikowska U, Ding M, Tan G, et al. Allergy. 2020;1-17. doi: https://doi.org/10.1111/all.14429

Background: Morbidity and mortality from COVID-19 caused by novel coronavirus SARSCoV-2 is accelerating worldwide, and novel clinical presentations of COVID-19 are often reported. The range of human cells and tissues targeted by
SARS-CoV-2, its potential receptors and associated regulating factors are still largely unknown. The aim of our study was to analyze the expression of known and potential SARS-CoV-2 receptors and related molecules in the extensive collection of primary human cells and tissues from healthy subjects of different age and from patients with risk factors and known comorbidities of COVID-19.

Methods: We performed RNA sequencing and explored available RNA-Seq databases to study gene expression and co-expression of ACE2, CD147 (BSG), and CD26 (DPP4) and their direct and indirect molecular partners in primary human bronchial epithelial cells, bronchial and skin biopsies, bronchoalveolar lavage fluid, whole blood, peripheral blood mononuclear cells (PBMCs), monocytes, neutrophils, DCs, NK cells, ILC1, ILC2, ILC3, CD4+ and $\mathrm{CD} 8+\mathrm{T}$ cells, $\mathrm{B}$ cells, and plasmablasts. We analyzed the material from healthy children and adults, and from adults in relation to their disease or COVID-19 risk factor status.

Results: ACE-2 and TMPRSS2 were co-expressed at the epithelial sites of the lung and skin, whereas CD 147 (BSG), cyclophilins (PPIA and PPIB), CD26 (DPP4), and related molecules were expressed in both epithelium and in immune cells. We also observed a distinct age-related expression profile of these genes in the PBMCs and $\mathrm{T}$ cells from healthy children and adults. Asthma, COPD, hypertension, smoking, obesity, and male gender status generally led to the higher expression of ACE2- and CD 147-related genes in the bronchial biopsy, BAL, or blood. Additionally, CD147-related genes correlated positively with age and BMI.

Conclusions: Our data suggest different receptor repertoire are potentially involved in the SARSCoV-2 infection at the epithelial barriers and in the immune cells. Altered expression of these receptors related to age, gender, obesity and smoking, as well as with disease status, might contribute to COVID-19 morbidity and severity patterns.

Keywords: COPD; COVID-19; COVID-19 children; SARS receptor; asthma; hypertension; obesity. 


\section{Comments}

The majority of individuals who become infected with COVID-19 have relatively mild symptoms however, approximately $20 \%$ of individuals demonstrate severe or critically severe disease such as pneumonia, respiratory failure, septic shock, multi-organ failure and thromboembolic disease. A majority of the deaths that have occurred from COVID-19 are in patients with chronic conditions such as cardiovascular disease, COPD, diabetes and obesity. Male gender and age are also identified as risk factors for more serious outcomes. The exact mechanisms for these observations remain unknown. It is hypothesized that COVID-19 may target specific organ systems secondary to factors that render patients with chronic disease more susceptible. Zhou et al recently reported that the SARS-CoV-2 uses the ACE-2 receptor as the cellular entry receptor. ${ }^{20}$ ACE-2 is responsible for the breakdown of angiotensin 2 to its metabolites that are potent vasodilators. ACE- 2 is expressed in a variety of different tissues including the upper and lower respiratory tract, myocardium and gastrointestinal mucosa. Leung et al has demonstrated that ACE-2 gene expression in bronchial epithelial cells was significantly increased in COPD versus non-COPD individuals (COPD 2.52 \pm 0.66 versus non-COPD 1.70 \pm 0.51$).{ }^{21}$ Current smokers were also shown to have a significantly higher gene expression of ACE-2 compared to never smokers, (current smokers $2.77 \pm 0.91$ versus never smokers $1.78 \pm 0.39$ $(P=0.024)$. Even when they adjusted for smoking status, the association between ACE-2 expression and COPD remained significant. The findings from this study further support the important role that the ACE-2 receptors may play in increased susceptibility in patients with COPD and other chronic conditions.

\section{Abstract 4 Risk of COVID-19-Related Death Among Patients with Chronic Obstructive Pulmonary Disease or Asthma Prescribed Inhaled Corticosteroids: An Observational Cohort Study Using the OpenSAFELY Platform}

Schultze A, Walker AJ, MacKenna B, et al. Lancet Respir Med. 2020; published online Sep 24, 2020.

doi: https://doi.org/10.1016/S2213-2600(20)30415-X

Background: Early descriptions of patients admitted to hospital during the COVID-19 pandemic showed a lower prevalence of asthma and chronic obstructive pulmonary disease (COPD) than would be expected for an acute respiratory disease like COVID-19, leading to speculation that inhaled corticosteroids (ICSs) might protect against infection with severe acute respiratory syndrome coronavirus 2 or the development of serious sequelae. We assessed the association between ICS and COVID-19-related death among people with COPD or asthma using linked electronic health records (EHRs) in England, UK.

Methods: In this observational study, we analyzed patient-level data for people with COPD or asthma from primary care EHRs linked with death data from the Office of National Statistics using the OpenSAFELY platform. The index date (start of follow-up) for both cohorts was March 1, 2020; follow-up lasted until May 6, 2020. For the COPD cohort, individuals were eligible if they were aged 35 years or older, had COPD, were a current or former smoker, and were prescribed an ICS or long-acting $\beta$ agonist plus long-acting muscarinic antagonist (LABA-LAMA) as combination therapy within the 4 months before the index date. For the asthma cohort, individuals were eligible if they were aged 18 years or older, had been diagnosed with asthma within 3 years of the index date, and were prescribed an ICS or short-acting $\beta$ agonist (SABA) only within the 4 months before the index date. We compared the outcome of COVID-19-related death between people prescribed an ICS and those prescribed alternative 
respiratory medications: ICSs versus LABA-LAMA for the COPD cohort, and low-dose or medium-dose and high-dose ICSs versus SABAs only in the asthma cohort. We used Cox regression models to estimate hazard ratios (HRs) and 95\% CIs for the association between exposure categories and the outcome in each population, adjusted for age, sex, and all other prespecified covariates. We calculated e-values to quantify the effect of unmeasured confounding on our results.

Findings: We identified 148557 people with COPD and 818490 people with asthma who were given relevant respiratory medications in the 4 months before the index date. People with COPD who were prescribed ICSs were at increased risk of COVID-19related death compared with those prescribed LABALAMA combinations (adjusted HR 1.39 [95\% CI 1.10-1.76]). Compared with those prescribed SABAs only, people with asthma who were prescribed highdose ICS were at an increased risk of death (1.55 [1.10-2.18]), whereas those given a low or medium dose were not $(1 \cdot 14[0 \cdot 85-1 \cdot 54])$. Sensitivity analyses showed that the apparent harmful association we observed could be explained by relatively small health differences between people prescribed ICS and those not prescribed ICS that were not recorded in the database (e value lower 95\% CI 1.43).

Interpretation: Our results do not support a major role for regular ICS use in protecting against COVID19-related death among people with asthma or COPD. Observed increased risks of COVID-19-related death can be plausibly explained by unmeasured confounding due to disease severity.

Funding: UK Medical Research Council.

\section{Comments}

This study demonstrated that while comorbidities were similar between groups, exacerbations in the previous year were more frequent in the ICS combination group suggesting that ICSs are used in patients with more frequent exacerbations and probable more severe disease. Unfortunately, the investigators did not include significant confounding factors in the analysis such as severity of COPD and exacerbation frequency.
Inclusion of such data would have helped clarify whether ICS use was the cause for worse outcomes related to COVID-19 or rather a surrogate marker of disease severity. Furthermore, in the COPD cohort there was an increased risk of COVID-related death for the ICS use group, even after adjusting for relevant factors such as age and comorbidities. A sensitivity analysis indicated that the ICS/long-acting beta2-agonist (LABA)/ long-acting muscarinic antagonists (LAMA) group were at the highest risk of death compared to LABA/LAMA and ICS/LABA groups. There were no significant differences in death rates comparing ICS/ LABA to LABA/LAMA. This would argue that the differences cannot be explained by ICS use. Rather, this would seem to indicate that patients who were on triple therapy had more severe underlying disease and thus were more likely to have poor outcomes. This study is instructive in highlighting the fact that ICS use may identify patients with more severe disease and represent a surrogate marker of underlying disease severity rather than being the cause for poorer outcomes. The results suggest that patients who are on ICSs should not necessarily stop them but that we should not try to put patients on higher doses of ICSs in hopes of them having a protective effect. 


\section{References}

1. Centers for Disease Control and Prevention (CDC). Coronavirus disease 2019. CDC COVID data tracker. CDC website. Published January 2020. Updated daily. Accessed October 11, 2020.

https://covid.cdc.gov/covid-data-tracker/\#cases_casesinlast7days

2. Johns Hopkins University and Medicine. Coronavirus disease resource center. Johns Hopkins website. Accessed October 11, 2020. https://coronavirus.jhu.edu/

3. Wu Z, McGoogan JM. Characteristics of and important lessons from the coronavirus disease 2019 (COVID-19) outbreak in China: summary of a report of 72314 cases from the Chinese Center for Disease Control and Prevention. JAMA. 2020;323(13):1239-1242.

doi: https://doi.org/10.1001/jama.2020.2648

4. Huang C, Wang Y, Li X, et al. Clinical features of patients infected with 2019 novel coronavirus in Wuhan, China. Lancet. 2020;395(10223): 497-506.

doi: https://doi.org/10.1016/S0140-6736(20)30183-5

5. Sin DD. COVID-19 in COPD: a growing concern. EClinicalMedicine. 2020; 26:100546.

doi: https://doi.org/10.1016/j.eclinm.2020.100546

6. Leung JM, Nikura M, Yang CWT, Sin DD. COVID-19 and COPD. Eur Respir J. 2020;56(2): 2002108.

doi: https://doi.org/10.1183/13993003.02108-2020

7. Guan WJ, Liang W, He J, Zhong N. Cardiovascular comorbidity and its impact on patients with COVID-19. Eur Respir J. 2020; 55(6):2001227.

doi: https://doi.org/10.1183/13993003.01227-2020

8. Guan WJ, Liang W, Zhao Y, et al. Comorbidity and its impact on 1590 patients with COVID-19 in China: a nationwide analysis. Eur Respir J. 2020; 55(5).

doi: https://doi.org/10.1183/13993003.00547-2020

9. Attaway AA, Zein J, Hatipoglu US. SARS-CoV-2 infection in the COPD population is associated with increased healthcare utilization: an analysis of Cleveland clinic's COVID-19 registry. EClinicalMedicine. 2020;26:100515.

doi: https://doi.org/10.1016/j.eclinm.2020.100515

10. Singanayagam A, Johnston SL. Long-term impact of inhaled corticosteroid use in asthma and chronic obstructive pulmonary disease (COPD): review of mechanisms that underlie risks. $J$ Allergy Clin Immunol. 2020; In press. doi: https://doi.org/10.1016/j.jaci.2019.12.907

11. Peters MC, Sajuthi S, Deford P, et al. COVID-19-related genes in sputum cells in asthma. Relationship to demographic features and corticosteroids. Am J Respir Crit Care Med. 2020;202(1):8390. doi: https://doi.org/10.1164/rccm.202003-0821OC
12. Yamaya M, Nishimura H, Deng X, et al. Inhibitory effects of glycopyrronium, formoterol, and budesonide on coronavirus HCoV-229E replication and cytokine production by primary cultures of human nasal and tracheal epithelial cells. Respir Investig. 2020;58(3):55-168. doi: https://doi.org/10.1016/j.resinv.2019.12.005

13. Dexamethasone for COVID-19: preliminary findings. Drug Ther Bull. 2020; 58(9):133. doi: https://doi.org/10.1136/dtb.2020.000045

14. RECOVERY Collaborative Group. Dexamethasone in hospitalized patients with COVID-19. Preliminary report. $N$ Engl J Med. 2020; published online July 17, 2020. doi: https://doi.org/ 10.1056/NEJMoa2021436

15. Boglione L, Rostagno R, Poletti F, et al. The proper use of corticosteroids for 2019-nCov pneumonia: towards promising results? J Infect. 2020;4781. doi: https://doi.org/10.1016/j.jinf.2020.08.001

16. $\mathrm{Hu} \mathrm{Z}, \mathrm{Lv} \mathrm{Y}, \mathrm{Xu} \mathrm{C}$, et al. Clinical use of short-course and low-dose corticosteroids in patients with non-severe COVID-19 during pneumonia progression. Front Public Health. 2020;8:355. doi: https://doi.org/10.3389/fpubh.2020.00355

17. Matthay MA, Wick KD. Corticosteroids, COVID-19 pneumonia and acute respiratory distress syndrome. J Clin Invest. 2020. doi: https://doi.org/10.1172/JCI143331

18. Zhang Q, Li S, Zhou W, Yang X, Li J, Cao J. Risk of pneumonia with different inhaled corticosteroids in COPD patients: a metasnalysis. COPD. 2020;17(4):462-469.

doi: https://doi.org/10.1080/15412555.2020.1787369

19. Halpin DMG, Singh D, Hadfield RM. Inhaled corticosteroids and COVID-19: a systematic review and clinical perspective. Eur Respir J. 2020;55(5).

doi: https://doi.org/10.1183/13993003.01009-2020

20. Zhou P, Yang X-L, Wang X-G, et al. A pneumonia outbreak associated with a new coronavirus of probable bat origin. Nature. 2020; 579(7798): 270-273.

doi: https://doi.org/10.1038/s41586-020-2012-7

21. Leung JM, Yang CX, Tam A, et al. ACE-2 expression in the small airway epithelia of smokers and COPD patients: implications for COVID-19. Eur Respir J. 2020; 55(5). doi: https://doi.org/10.1183/13993003.00688-2020 